TRANSACTIONS OF THE

AMERICAN MATHEMATICAL SOCIETY

Volume 356, Number 12, Pages 4687-4700

S 0002-9947(04)03590-1

Article electronically published on June 22, 2004

\title{
EXPANSIVENESS OF ALGEBRAIC ACTIONS ON CONNECTED GROUPS
}

\author{
SIDDHARTHA BHATTACHARYA
}

\begin{abstract}
We study endomorphism actions of a discrete semigroup $\Gamma$ on a connected group $G$. We give a necessary and sufficient condition for expansiveness of such actions provided $G$ is either a Lie group or a solenoid.
\end{abstract}

\section{INTRODUCTION}

If $X$ is a topological group and $\rho$ is an action of a semigroup $\Gamma$ on $X$ by continuous endomorphisms of $X$, then $\rho$ is said to be expansive if there exists a neighborhood $U$ of the identity in $X$ such that

$$
\bigcap_{\gamma} \rho(\gamma)^{-1}(U)=\{e\} .
$$

Any such neighborhood is called an expansive neighborhood of the identity in $X$. An automorphism $\tau$ of $X$ is said to be expansive if the cyclic group generated by $\tau$ acts expansively on $X$. It is easy to check that when $X$ is compact and metrizable this coincides with the metric definition of expansiveness.

The notion of expansiveness plays an important role in the study of endomorphism actions. Earlier, expansiveness of automorphisms of connected groups was studied by several authors. A complete description of expansive automorphisms on compact connected groups was obtained in [10]. In particular, it was shown that every compact connected group which admits an expansive automorphism is abelian and finite-dimensional.

In recent years dynamics of endomorphism actions of discrete semigroups on compact abelian groups has been extensively studied using techniques from commutative algebra (cf. [14). Among other things, a complete characterization of expansiveness has been obtained when $X$ is zero-dimensional or $\Gamma=\mathbb{Z}^{d}$ for some $d \geq 1$ (cf. 13], Theorem 3.9). For further study of expansiveness and its relation with other dynamical properties the reader is referred to [3], 6] and [11].

In this paper we consider expansive endomorphism actions of arbitrary semigroups on connected metrizable topological groups. For any such action $(G, \rho)$ we give necessary and sufficient conditions for expansiveness of $\rho$, provided $G$ is either a Lie group or a compact finite-dimensional group.

This paper is organized as follows. In section 2 we prove some elementary results about expansiveness of endomorphism actions on finite-dimensional vector spaces, considered as abelian topological groups under addition. In Section 3 we consider

Received by the editors September 6, 2000 and, in revised form, April 19, 2001.

2000 Mathematics Subject Classification. Primary 37B05; Secondary 54H20.

Key words and phrases. Expansive action, Lie group, solenoid.

(C)2004 American Mathematical Society 
endomorphism actions on connected Lie groups. If $G$ is a connected Lie group, then by $L(G)$ we denote the Lie algebra of $G$. If $\rho$ is an endomorphism action of a semigroup $\Gamma$ on $G$, then by $\rho_{e}$ we denote the induced $\Gamma$-action on $L(G)$ defined by

$$
\rho_{e}(\gamma)=\left.\mathrm{d} \rho(\gamma)\right|_{e} \forall \gamma \in \Gamma
$$

We prove the following.

Theorem A. Let $G$ be a connected Lie group, $\Gamma$ be a discrete semigroup and $\rho$ be an endomorphism action of $\Gamma$ on $G$. Then $(G, \rho)$ is expansive if and only if for all non-zero $v$ in $L(G)$, the $\rho_{e}$-orbit of $v$ is unbounded.

In the special case when $\Gamma$ is either abelian or a virtually nilpotent group, applying the above theorem we give a necessary and sufficient condition for expansiveness of $(G, \rho)$ in terms of the generalized weights of $\rho_{e}$. As a consequence we prove that if $\Gamma$ is a virtually nilpotent group and $(G, \rho)$ is expansive, then $\rho(\Gamma)$ contains an expansive automorphism of $G$.

In section 4 we consider endomorphism actions on compact connected finitedimensional abelian groups. For any such group $G$, by $\widehat{G}$ we denote the Pontryagin dual of $G$ and by $L(G)$ we denote the vector space of all homomorphisms from $\widehat{G}$ to $\mathbb{R}$ under pointwise addition and scalar multiplication. From the duality theory of compact abelian groups it follows that $L(G)$ is finite-dimensional. If $\rho$ is an endomorphism action of a semigroup $\Gamma$ on $G$, then by $\rho_{e}$ we denote the induced $\Gamma$-action on $L(G)$ defined by

$$
\rho_{e}(\gamma)(p)(\chi)=p(\chi \circ \rho(\gamma)) \quad \forall p \in L(G) .
$$

Also, for such an action $\rho$, by $\widehat{\rho}$ we denote the induced $\Gamma$-action on $\widehat{G}$. We note that $\widehat{G}$ can be realised as a module over $\mathbb{Z}(\Gamma)$, the group-ring of $\Gamma$, via the action $\widehat{\rho}$.

We prove the following.

Theorem B. Let $\Gamma$ be a discrete semigroup and let $\rho$ be an endomorphism action of $\Gamma$ on a compact connected finite-dimensional abelian group $G$. Then $(G, \rho)$ is expansive if and only if the following two conditions are satisfied:

a) $\widehat{G}$ is finitely generated as a $\mathbb{Z}[\Gamma]$-module.

b) For every non-zero $v$ in $L(G)$, the $\rho_{e}$-orbit of $v$ is unbounded.

It is known that non-abelian compact connected groups do not admit expansive endomorphism actions (cf. 99). Therefore Theorem B characterizes expansive endomorphism actions on arbitrary compact connected finite-dimensional groups.

\section{Preliminaries}

Throughout this paper $\Gamma$ will denote a discrete semigroup i.e. a set with an associative multiplication law. If $\Gamma_{1}, \Gamma_{2}$ are semigroups, then a map $f$ from $\Gamma_{1}$ to $\Gamma_{2}$ is said to be a semigroup homomorphism if for all $\gamma_{1}, \gamma_{2}$ in $\Gamma_{1}, f\left(\gamma_{1} \cdot \gamma_{2}\right)=$ $f\left(\gamma_{1}\right) \cdot f\left(\gamma_{2}\right)$. If $G$ is a topological group, then by $\operatorname{End}(G)$ we denote the semigroup of all continuous endomorphisms of $G$, with composition as the product law.

Suppose $G$ is a topological group and $\rho: \Gamma \rightarrow \operatorname{End}(G)$ is an endomorphism action of a discrete semigroup $\Gamma$ on $G$. Then it is easy to see that $\Gamma_{1}=\rho(\Gamma) \cup\{\operatorname{Id}\}$ is a subsemigroup of $\operatorname{End}(G)$ and $\rho$ is expansive if and only if the natural action of $\Gamma_{1}$ on $G$ is expansive. Henceforth we will restrict the discussion of endomorphism 
actions to the case when $\Gamma$ contains an identity element $e$ and $\rho(e) \in \operatorname{End}(G)$ is the identity automorphism.

If $\mathbf{C}$ denotes the semigroup of complex numbers under multiplication, then any semigroup homomorphism $\lambda: \Gamma \rightarrow \mathbf{C}$ is said to be a character of $\Gamma$. If $\rho: \Gamma \rightarrow$ $\operatorname{End}(V)$ is an endomorphism action of $\Gamma$ on a finite-dimensional vector space $V$ over $\mathbb{C}$, then $\rho$ is said to be reducible if there exists characters $\lambda_{1}, \ldots, \lambda_{k}$ and non-trivial $\rho$-invariant subspaces $V_{1}, \ldots, V_{k} \subset V$ satisfying the following conditions:

1) $V=V_{1} \oplus \cdots \oplus V_{k}$.

2) For $i=1, \ldots, k$, there exists a basis $B$ of $V_{i}$ such that $\left.\rho(\gamma)\right|_{V_{i}}-\lambda_{i}(\gamma) I$ is strictly upper triangular with respect to $B$, for all $\gamma$ in $\Gamma$.

The characters $\lambda_{1}, \ldots, \lambda_{k}$ are said to be the generalized weights of $\rho$ and for all $i$, $V_{i}$ is said to be the generalized weight space corresponding to $\lambda_{i}$.

Example. If $\rho(\Gamma)$ is an abelian subsemigroup of $\operatorname{End}(V)$, then $\rho$ is reducible (see [5], pg. 134). This is also true when $\rho(\Gamma)$ is contained in a connected nilpotent subgroup $H$ of $G L(V)$. This can be seen by looking at the Lie algebra homomorphism $i: L(H) \rightarrow \operatorname{End}(V)$ and using certain fundamental facts about representations of nilpotent Lie algebras (see [8], Proposition 2.4 and Theorem 1.35).

The following proposition gives a necessary and sufficient condition for expansiveness of endomorphism actions on a finite-dimensional vector space, considered as an abelian group under addition.

Proposition 2.1. Let $\Gamma$ be a discrete semigroup and let $\rho: \Gamma \rightarrow \operatorname{End}(V)$ be an endomorphism action of $\Gamma$ on a finite-dimensional vector space $V$ over $\mathbb{R}$ or $\mathbb{C}$. Then we have the following:

a) $\rho$ is expansive if and only if for all non-zero $v$ in $V$, the $\Gamma$-orbit of $v$ is unbounded.

b) If $V$ is a real vector space, then $\rho$ is expansive if and only if the induced endomorphism action of $\Gamma$ on $V \otimes \mathbb{C}$ is expansive.

Proof. For any $v$ in $V$ let $O_{v} \subset V$ denote the $\rho$-orbit of $v$. Suppose that there is a non-zero vector $v$ in $V$ with bounded $\rho$-orbit. Let $U$ be any neighborhood of 0 in $V$. Then for $\epsilon>0$ sufficiently small, $O_{\epsilon v}=\epsilon O_{v}$ is contained in $U$. This implies that $\rho$ is not expansive. On the other hand if $O_{v}$ is unbounded for all non-zero $v \in V$, then it is easy to see that any bounded neighborhood of 0 is an expansive neighborhood. This proves a). Part b) is an immediate consequence of a).

Proposition 2.2. Let $\Gamma$ be a discrete semigroup, $V$ be a finite-dimensional vector space over $\mathbb{C}$ and $\rho: \Gamma \rightarrow \operatorname{End}(V)$ be a reducible endomorphism action with generalized weights $\lambda_{1}, \ldots, \lambda_{k}$. Then $\rho$ is expansive if and only if for each $i$, the image of $\lambda_{i}$ is an unbounded subset of $\mathbb{C}$.

Proof. For $i=1, \ldots, k$ let $V_{i}$ be the generalized weight space corresponding to $\lambda_{i}$. Since each $V_{i}$ is $\rho$-invariant, from the previous proposition it follows that $\rho$ is expansive if and only if $\left.\rho\right|_{V_{i}}$ is expansive for all $i$. Therefore without loss of generality we may assume that $k=1$. In that case there exists a character $\lambda$ of $\Gamma$ such that after suitable identifications we have:

a) $V=\mathbb{C}^{n}$ for some $n>0$ and 
b) for all $\gamma \in \Gamma, \rho(\gamma)$ can be written as,

$$
\rho(\gamma)=\left(\begin{array}{ccccc}
\lambda(\gamma) & & & & \\
& \ddots & & * & \\
& & \lambda(\gamma) & & \\
& 0 & & \ddots & \\
& & & & \lambda(\gamma)
\end{array}\right)
$$

Suppose there exists a non-zero vector $v=\left(v_{1} \ldots, v_{n}\right)^{t}$ in $\mathbb{C}^{n}$ such that the $\rho$-orbit of $v$ is bounded. Let $j \leq n$ be the largest positive integer such that $v_{j} \neq 0$. Then it is easy to see that

$$
\left\{\rho(\gamma)(v)_{j} \mid \gamma \in \Gamma\right\}=\left\{\lambda(\gamma) v_{j} \mid \gamma \in \Gamma\right\} .
$$

Since the $\rho$-orbit of $v$ is bounded, this implies that the image of $\lambda$ is a bounded subset of $\mathbb{C}$. Conversely, it is easy to see that if $v \in \mathbb{C}^{n}$ denotes the vector $(1,0, \ldots, 0)^{t}$, then

$$
\{\rho(\gamma)(v) \mid \gamma \in \Gamma\}=\left\{(\lambda(\gamma), 0, \ldots, 0)^{t} \mid \gamma \in \Gamma\right\} .
$$

Therefore if the image of $\lambda$ is bounded, then there exists a non-zero vector in $V$ with bounded $\rho$-orbit.

In the special case when $\Gamma$ is isomorphic to $\mathbb{Z}^{+}$or $\mathbb{Z}$ we obtain the following consequence of the above proposition. This was first proved in [4].

Proposition 2.3. Let $V$ be a finite-dimensional vector space over $\mathbb{R}$ or $\mathbb{C}$ and let $T$ be an element of End $(V)$. Then the semigroup composed of positive powers of $T$ acts expansively on $V$ if and only if the spectrum of $T$ does not intersect the closed unit disk. If $T$ is invertible, then the cyclic subgroup generated by $T$ acts expansively on $V$ if and only if the spectrum of $T$ does not intersect $\mathbb{S}^{1}$.

Proof. Replacing $V$ by $V \otimes \mathbb{C}$ if necessary, we may assume that $V$ is a complex vector space. Let $\Gamma=\left\{T^{n} \mid n \geq 0\right\}$ denote the cyclic semigroup generated by $T$ and let $\rho$ denote the natural action of $\Gamma$ on $V$. Since $\Gamma$ is abelian, $\rho$ is reducible. Furthermore if $\lambda_{1}, \ldots, \lambda_{k}$ denote the generalized weights of $\rho$, then $\lambda_{1}(T), \ldots, \lambda_{k}(T)$ are the eigenvalues of $T$. Applying the previous proposition we conclude that $\rho$ is expansive if and only if the spectrum of $T$ does not intersect the closed unit disk. The second assertion follows from a similar argument.

\section{EXPANSIVE ACTIONS ON LiE GROUPS}

In this section we prove Theorem $\mathrm{A}$ and its consequences. Throughout the section $G$ will denote a connected Lie group and $L(G)$ will denote the Lie algebra of $G$. The standard exponential map from $L(G)$ to $G$ will be denoted by exp.

We begin with the following lemma.

Lemma 3.1. Let $V$ be a finite-dimensional vector space over $\mathbb{R}$ and let $\rho$ be an expansive endomorphism action of a discrete semigroup $\Gamma$ on $V$. Then there exists a finitely generated subsemigroup $\Gamma_{0} \subset \Gamma$ which acts expansively on $V$.

Proof. Suppose this is not the case. We fix a norm $\|$.$\| on V$. Let $S \subset V$ denote the set defined by $S=\{v \in V \mid\|v\|=1\}$. For every finitely generated subsemigroup $\Lambda \subset \Gamma$ we define a closed subset $S(\Lambda) \subset S$ by

$$
S(\Lambda)=\{v \in S \mid\|\rho(\gamma)(v)\| \leq 1 \forall \gamma \in \Lambda\} .
$$


We claim that for any finitely generated subsemigroup $\Lambda \subset \Gamma, S(\Lambda)$ is non-empty. To see this we define a $\Lambda$-invariant subspace $V(\Lambda) \subset V$ by

$$
V(\Lambda)=\{v \in V \mid \Lambda-\text { orbit of } v \text { is bounded }\} .
$$

By Proposition 2.1, $V(\Lambda)$ is a non-trivial subspace of $V$. Let $\Lambda_{b} \subset \operatorname{End}(V(\Lambda))$ be the image of $\Lambda$ under the map $\left.\gamma \mapsto \rho(\gamma)\right|_{V(\Lambda)}$. Fix a basis $B=\left\{v_{1}, \ldots, v_{k}\right\}$ of $V(\Lambda)$ and identify $\Lambda_{b}$ with a subsemigroup $K$ of $M(k, \mathbb{R})$. Since for any $v \in V(\Lambda)$ the $\Lambda_{b}$-orbit of $v$ is a bounded subset of $V(\Lambda)$, it follows that for all $1 \leq i, j \leq k$, the $(i, j)$ th entries of $K$ lie in a bounded subset of $\mathbb{R}$. This shows that $\overline{\Lambda_{b}}$ is a compact subsemigroup of $\operatorname{End}(V(\Lambda))$. We choose a non-zero $w \in V(\Lambda)$ and define a continuous function $h: \overline{\Lambda_{b}} \rightarrow \mathbb{R}$ by $h(\alpha)=\|\alpha(w)\|$ for all $\alpha$ in $\overline{\Lambda_{b}}$. Since $\overline{\Lambda_{b}}$ is compact, there exists a $\alpha_{0} \in \overline{\Lambda_{b}}$ such that $h\left(\alpha_{0}\right) \geq h(\alpha)$ for all $\alpha$ in $\overline{\Lambda_{b}}$. Putting $v=\alpha_{0}(w)$ we see that for any $\alpha$ in $\overline{\Lambda_{b}}$,

$$
\|\alpha(v)\|=h\left(\alpha \alpha_{0}\right) \leq h\left(\alpha_{0}\right)=\|v\| .
$$

Since $\overline{\Lambda_{b}}$ contains Id, $\left\|\alpha_{0}(w)\right\| \geq\|w\|>0$. Now it is easy to see that the unit vector $v /\|v\| \in S(\Lambda)$, which proves the claim.

If $\Lambda_{1}, \Lambda_{2}, \ldots, \Lambda_{k}$ are finitely generated subsemigroups of $\Gamma$ and $\Lambda$ is a finitely generated subsemigroup of $\Gamma$ containing $\Lambda_{1}, \Lambda_{2}, \ldots, \Lambda_{k}$, then from the above claim it follows that

$$
\emptyset \neq S(\Lambda) \subset S\left(\Lambda_{1}\right) \cap \cdots \cap S\left(\Lambda_{k}\right) .
$$

This shows that the collection $\{S(\Lambda)\}$ has the finite-intersection property. Now from the compactness of $S$ it follows that

$$
\bigcap_{\Lambda} S(\Lambda) \neq \emptyset
$$

Clearly for any vector $w$ which lies in the intersection, the $\Gamma$-orbit of $w$ is bounded, which contradicts our hypothesis.

Proof of Theorem A. Suppose $(G, \rho)$ is expansive. Let $U$ be an expansive neighborhood of the identity in $G$. We choose a neighborhood $V$ of 0 in $L(G)$ such that $\left.\exp \right|_{V}$ is a homeomorphism and $\exp (V)$ is contained in $U$. Let $v$ be any non-zero element of $L(G)$ such that the $\rho_{e}$-orbit of $v$ is contained in $V$. Since exp is a $\Gamma$ equivariant map from $\left(L(G), \rho_{e}\right)$ to $(G, \rho)$, it follows that the $\rho$-orbit of $\exp (v)$ is contained in $U$. Since $U$ is an expansive neighborhood of the identity and $\left.\exp \right|_{V}$ is a homeomorphism, this implies that $v=0$. Hence $\rho_{e}$ is expansive. Now applying Proposition [2.1] we see that for every non-zero $v$ in $L(G)$, the $\rho_{e}$-orbit of $v$ is unbounded.

Now we will prove the converse. Applying Lemma 3.1 and Proposition 2.1 we see that it is enough to consider the case when $\Gamma$ is finitely generated. Let $A$ be a finite set which generates $\Gamma$. We choose a neighborhood $V$ of 0 in $L(G)$ such that $\left.\exp \right|_{V}$ is a homeomorphism and $\bar{V}$ is compact. We choose another neighborhood $U$ of 0 such that

$$
U \subset V \text { and } \rho_{e}(\gamma)(U) \subset V \forall \gamma \in A
$$

We claim that $\exp (U)$ is an expansive neighborhood of $(G, \rho)$. Suppose this is not the case. Let $g \neq e$ be any element of $G$ such that the $\rho$-orbit of $g$ is contained in $\exp (U)$. We choose $v$ in $U$ such that $\exp (v)=g$. Let $O_{v}$ be the $\rho_{e}$-orbit of $v$. Since 
$v$ is non-zero, $O_{v}$ is unbounded. Since $A$ generates $\Gamma$ and $v \in U$, it follows that there exists $w$ in $O_{v}$ and $\gamma_{0} \in A$ such that

$$
w \in U \text { and } \rho_{e}\left(\gamma_{0}\right)(w) \in V-U .
$$

Since exp maps the $\rho_{e}$-orbit of $v$ onto the $\rho$-orbit of $\exp (v)$, this implies that the $\rho$-orbit of $\exp (v)$ intersects $\exp (V-U)$. Since $\exp (V-U)$ and $\exp (U)$ are disjoint, this gives a contradiction.

We note the following consequence of Theorem A and Lemma 3.1.

Corollary 3.2. Let $\Gamma$ be a discrete semigroup, $G$ be a connected Lie group and $\rho$ be an expansive endomorphism action of $\Gamma$ on $G$. Then there exists a finitely generated subsemigroup $\Gamma_{0} \subset \Gamma$ such that the action of $\rho$, restricted to $\Gamma_{0}$, is expansive.

The following corollary provides a rich source of expansive endomorphism actions of non-abelian semigroups on tori.

Corollary 3.3. Let $\Gamma$ be an infinite subsemigroup of $M(n, \mathbb{Z})$ which acts irreducibly on $\mathbb{R}^{n}$. Then the induced $\Gamma$-action on $\mathbb{T}^{n} \cong \mathbb{R}^{n} / \mathbb{Z}^{n}$ is expansive.

Proof. Let $\rho$ denote the natural action of $\Gamma$ on $\mathbb{R}^{n}$ and let $W$ denote the subspace of $\mathbb{R}^{n}$ consisting of all points with bounded $\rho$-orbit. Since $\Gamma$ is an infinite subsemigroup of $M(n, \mathbb{Z})$, it is a non-compact subset of of $M(n, \mathbb{R})$. Hence $W$ is a proper subspace of $\mathbb{R}^{n}$. From irreducibility of $\rho$ we conclude that $W=\{0\}$. Now applying Theorem A we see that the action of $\Gamma$ on $\mathbb{T}^{n}$ is expansive.

Corollary 3.4. Let $G$ be a connected Lie group and let $\rho$ be an expansive automorphism action of a virtually nilpotent group $\Gamma$ on $G$. Then $\rho(\Gamma)$ contains an expansive automorphism of $G$.

Proof. Since $\Gamma$ acts expansively on $G$, so does every finite-index subgroup of $\Gamma$. Therefore without loss of generality we may assume that $\Gamma$ is nilpotent. First we will consider the case when $G$ is isomorphic to a finite-dimensional vector $V$ space over $\mathbb{C}$. Let $H \subset G L(V)$ be the Zariski-closure of $\rho(\Gamma)$ and let $H_{0}$ be the connected component of $H$ which contains the identity. Since $H$ has finitely many components, it follows that

$$
\Gamma_{0}=\left\{\gamma \in \Gamma \mid \rho(\gamma) \in H_{0}\right\}
$$

is a finite-index subgroup of $\Gamma$. Let $\rho_{0}$ denote the $\Gamma_{0}$-action on $V$, induced by $\rho$. Since $\rho\left(\Gamma_{0}\right)$ is contained in a connected nilpotent subgroup of $G L(V), \rho_{0}$ is reducible. Since $\rho$ is expansive, so is $\rho_{0}$. Let $\lambda_{1}, \ldots, \lambda_{k}$ be the generalized weights of $\rho_{0}$. For each $\gamma$ in $\Gamma_{0}$ we define $A_{\gamma} \subset\{1, \ldots, k\}$ by

$$
A_{\gamma}=\left\{j|| \lambda_{i}(\gamma) \mid \neq 1\right\} .
$$

For any $\gamma \in \Gamma_{0}$ let $n(\gamma)$ denote the cardinality of $A_{\gamma}$. We choose $\gamma_{0} \in \Gamma_{0}$ such that the $n\left(\gamma_{0}\right) \geq n(\gamma)$ for all $\gamma$ in $\Gamma_{0}$. We claim that $n\left(\gamma_{0}\right)=k$ i.e. $\left|\lambda_{i}\left(\gamma_{0}\right)\right| \neq 1$ for all $j=1, \ldots, k$. Suppose this is not the case. We choose $i$ such that $\left|\lambda_{i}\left(\gamma_{0}\right)\right|=1$. By Proposition 2.2 there exists $\gamma_{1}$ such that $\left|\lambda_{i}\left(\gamma_{1}\right)\right| \neq 1$. It is easy to see that for sufficiently large $m>0$,

$$
\left|\lambda_{j}\left(\gamma_{0}^{m} \gamma_{1}\right)\right| \neq 1 \forall j \in A_{\gamma_{0}} \cup\{i\} .
$$

Since $n\left(\gamma_{0}\right) \geq n(\gamma)$ for all $\gamma$ in $\Gamma_{0}$, this gives a contradiction. Since the numbers $\lambda_{1}\left(\gamma_{0}\right), \ldots, \lambda_{k}\left(\gamma_{0}\right)$ are the eigenvalues of $\rho\left(\gamma_{0}\right)$, from the above claim and Proposition 2.3 we see that $\rho\left(\gamma_{0}\right)$ is an expansive automorphism of $V$. 
Now we will consider the general case. Let $\sigma$ be the $\Gamma$-action on the complexified Lie algebra $L(G) \otimes \mathbb{C}$, induced by $\rho_{e}$. Applying Theorem A and Proposition 2.1 we see that $\sigma$ is expansive. By the previous argument there exists a $\gamma_{0}$ in $\Gamma$ such that $\sigma\left(\gamma_{0}\right)$ is an expansive automorphism of $L(G) \otimes \mathbb{C}$. Applying Theorem A and Proposition 2.1 to the cyclic group generated by $\gamma_{0}$, we conclude that $\rho\left(\gamma_{0}\right)$ is an expansive automorphism of $G$.

As mentioned in the Introduction, non-abelian compact connected groups do not admit expansive endomorphism actions. The following example shows that this is not true if the group is not assumed to be compact.

Example. Fix $n \geq 3$ and positive real numbers $c_{1}<c_{2}<\cdots<c_{n}$. Let $D$ denote the diagonal element in $\operatorname{GL}(n, \mathbb{R})$ with diagonal entries $c_{1}, c_{2}, \ldots, c_{n}$. Let $G \subset \operatorname{GL}(n, \mathbb{R})$ denote the subgroup consisting of all unipotent upper triangular matrices and let $T$ denote the automorphism of $G$ defined by $T(X)=D^{-1} X D$. Let $\Gamma$ be the semigroup consisting of positive powers of $T$. For any $X \in G, X \neq \mathrm{Id}$, choose $1 \leq i<j \leq n$ such that $X_{i j} \neq 0$. Note that

$$
\left|T^{k}(X)_{i j}\right|=\left(c_{i}^{-1} c_{j}\right)^{k}\left|X_{i j}\right| \rightarrow \infty \text { as } k \rightarrow \infty .
$$

Therefore for any bounded neighborhood $U \subset G$ of the identity, the $\Gamma$-orbit of $X$ intersects $G-U$. Hence $U$ is an expansive neighbourhood for the natural action of $\Gamma$ on $G$.

We conclude this section with an example showing that Corollary 3.4 does not hold for actions of arbitrary discrete groups.

Example. Fix $n \geq 3$ and define a subgroup $\Gamma$ of $G L(n, \mathbb{R})$ by

$$
\Gamma=\left\{\left(\begin{array}{cc}
A & b \\
0 & 1
\end{array}\right) \mid A \in G L(n-1, \mathbb{Z}), b \in \mathbb{Z}^{n-1}\right\} .
$$

It is easy to see that for any $x=\left(x_{1}, \ldots, x_{n}\right)$ in $\mathbb{R}^{n}$, the $\Gamma$-orbit of $x$ is given by the set

$$
\left\{\left(\begin{array}{c}
A y+x_{n} b \\
x_{n}
\end{array}\right) \mid A \in G L(n-1, \mathbb{Z}), b \in \mathbb{Z}^{n-1}\right\} ; y=\left(x_{1}, \ldots, x_{n-1}\right) .
$$

Hence for every non-zero $x$ in $\mathbb{R}^{n}$, the $\Gamma$-orbit of $x$ is unbounded. Since the spectrum of $\gamma$ contains 1 for every $\gamma$ in $\Gamma$, applying Proposition 2.3 we see that $\Gamma$ does not contain any expansive automorphism of $\mathbb{R}^{n}$.

\section{EXPANSIVE ACTIONS ON SOLENOIDS}

In this section we consider endomorphism actions on solenoids (compact connected finite-dimensional abelian groups). We freely use various results from duality theory of locally compact abelian groups; the reader is referred to [12] for details.

For any locally compact abelian group $G$, we denote by $\widehat{G}$ the dual group of $G$. Recall that for a compact connected abelian group $G$, we denote by $L(G)$ the vector space consisting of all homomorphisms from $\widehat{G}$ to $\mathbb{R}$, under pointwise addition and scalar multiplication. It is known that if $G$ is a solenoid, then $\widehat{G}$ is a torsion free discrete abelian group of finite rank. Hence for any solenoid $G, L(G)$ is finite-dimensional. 
If $\rho$ is an endomorphism action of a semigroup $\Gamma$ on a compact connected abelian group $G$, then by $\widehat{\rho}$ we denote the induced $\Gamma$-action on $\widehat{G}$ and by $\rho_{e}$ we denote the induced $\Gamma$-action on $L(G)$ defined by

$$
\rho_{e}(\gamma)(p)(\chi)=p(\chi \circ \rho(\gamma)) .
$$

Example. Let $\Gamma$ be a semigroup, $\widehat{\rho}: \Gamma \rightarrow M(n, \mathbb{Q})$ be a semigroup homomorphism for some $n \geq 1$ and $H$ be a $\Gamma$-invariant subgroup of $\mathbb{Q}^{n}$. Then the action of $\Gamma$ on $H$ induces an endomorphism action $\rho$ of $\Gamma$ on $G=\widehat{H}$. If $H$ contains $\mathbb{Z}^{n}$, then $L(G)$ can be identified with $\mathbb{R}^{n}$ and $\rho_{e}$ can be identified with the adjoint of the $\Gamma$-action on $\mathbb{R}^{n}$ induced by $\widehat{\rho}$.

This section is organized as follows. Throughout this section $G$ will denote a solenoid and $\rho$ will denote an endomorphism action of a discrete semigroup $\Gamma$ on $G$. In Proposition 4.1 we prove that the conditions a) and b), as stated in Theorem B, are necessary for expansiveness of $\rho$. In Proposition 4.2 we show that expansiveness of $\rho$ and existence of non-trivial bounded $\rho_{e}$-orbit in $L(G)$ can be characterized in terms of suitably chosen metrics on $G$ and $L(G)$, respectively. In Proposition 4.3 we prove Theorem B when $\Gamma$ is finitely generated. We complete the proof of Theorem $\mathrm{B}$ in Theorem 4.4 .

4.1. Necessary conditions for expansiveness. If $G$ is a compact connected abelian group, then we define a map $E$ from $L(G)$ to $G$ by the condition

$$
(\phi \circ E)(p)=e^{2 \pi i p(\phi)} \quad \forall p \in L(G), \phi \in \widehat{G} .
$$

Since for a fixed $p$ in $L(G)$, the map $\phi \rightarrow e^{2 \pi i p(\phi)}$ is a continuous homomorphism from $\widehat{G}$ to $\mathbb{S}^{1}$, by the duality theorem the map $E$ is well defined and unique. From the uniqueness it follows that $E$ is a homomorphism from $L(G)$ (considered as an abelian group under addition) to $G$. It is easy to check that the kernel of $E$ can be identified with the set of all homomorphisms from $\widehat{G}$ to $\mathbb{Z}$, which is a discrete subgroup of $L(G)$.

Remark. Using the duality theorem we can realize $L(G)$ with the set of all oneparameter subgroups of $G$ and $E$ with the map $\alpha \mapsto \alpha(1)$. In particular when $G$ is a torus, $L(G)$ can be identified with $\mathbb{R}^{n}$, the Lie algebra of $G$, and $E$ can be identified with the standard exponential map. However in general the map $E$ is not surjective.

Definition. Let $\Gamma$ be a semigroup and let $\rho$ be an endomorphism action of $\Gamma$ on a compact abelian group $G$. Then a set $A \subset \widehat{G}$ is said to be a $\rho$-basis if $A$ generates $\widehat{G}$ as an abelian group and $A=\bigcup_{\gamma} \widehat{\rho}(\gamma)(F)$ for some finite set $F \subset \widehat{G}$.

Clearly $\widehat{G}$ admits a $\rho$-basis if and only if it is finitely generated as a $\mathbb{Z}(\Gamma)$-module.

The next two propositions show that for expansive endomorphism actions the conditions a) and b), as stated in Theorem B, are satisfied. The results are known; we include the proofs for the sake of completeness.

Proposition 4.1. Let $\Gamma$ be a discrete semigroup and let $\rho$ be an expansive endomorphism action of $\Gamma$ on a compact abelian group $G$. Then $\widehat{G}$ has a $\rho$-basis. 
Proof. Let $U$ be an expansive neighborhood of of $e$ in $G$. We choose a finite set $F \subset \widehat{G}$ and $\epsilon>0$ such that

$$
\bigcap_{\chi \in F}\{g \in G|| \chi(g)-1 \mid<\epsilon\} \subset U .
$$

We define $A \subset \widehat{G}$ by $A=\bigcup_{\gamma} \widehat{\rho}(\gamma)(F)$. Let $\widehat{H} \subset \widehat{G}$ be the subgroup generated by $A$ and let $G^{\prime} \subset G$ be the subgroup consisting of all $g$ in $G$ such that $\chi(g)=e$ for all $\chi$ in $\widehat{H}$. Then for every $g \in G^{\prime}$, the $\rho$-orbit of $g$ is contained in $U$. Since $U$ is an expansive neighborhood of of $e$, we conclude that $G^{\prime}=\{e\}$. Now from duality theory of compact abelian groups it follows that $\widehat{H}=\widehat{G}$ (see [12], Theorem 53). Hence $A$ is a $\rho$-basis of $\widehat{G}$.

Proposition 4.2 (See also [2, proof of Corollary 1). Let $\Gamma$ be a discrete semigroup and let $\rho$ be an expansive endomorphism action of $\Gamma$ on a solenoid $G$. Then for every non-zero point $p \in L(G)$, the orbit of $p$ under $\rho_{e}$ is unbounded.

Proof. Let $U$ be an expansive neighborhood of $e$ in $G$. Since $E$ is a $\Gamma$-equivariant map from $\left(L(G), \rho_{e}\right)$ to $(G, \rho)$, it follows that

$$
\bigcap_{\gamma \in \Gamma} \rho_{e}(\gamma)^{-1}\left(E^{-1}(U)\right) \subset E^{-1}\left(\bigcap_{\gamma \in \Gamma} \rho(\gamma)^{-1}(U)\right)=\operatorname{Ker}(E) .
$$

Since the kernel of $E$ is discrete, there exists an open set $V \subset E^{-1}(U)$ such that $V \cap \operatorname{ker}(E)=\{0\}$. From the above identity it is easy to see that $V$ is an expansive neighborhood of 0 for the action $\rho_{e}$. Now the given assertion follows from Proposition 2.1.

4.2. Metrics induced by endomorphism actions. Let $\delta$ be the function from $\mathbb{S}^{1}$ to $\mathbb{R}$ defined by $\delta(z)=\inf \left\{|t| \mid t \in \mathbb{R}, e^{2 \pi i t}=z\right\}$. For any solenoid $G$ and $A \subset \widehat{G}$ we define functions $d_{A}: G \times G \rightarrow[0,1]$ and $d_{A}^{*}: L(G) \times L(G) \rightarrow[0, \infty]$ by

$$
\begin{aligned}
& d_{A}(g, h)=\sup _{\chi \in A} \delta \circ \chi\left(g^{-1} h\right), \\
& d_{A}^{*}(p, q)=\sup _{\chi \in A}|p(\chi)-q(\chi)| .
\end{aligned}
$$

It is easy to see that if $A$ generates $\widehat{G}$ as an abelian group, then $d_{A}$ is a metric on $G$ and $d_{A}^{*}$ is a metric on the subspace $L(G)_{A} \subset L(G)$ consisting of all $p$ in $L(G)$ with $d_{A}^{*}(0, p)<\infty$. For $A \subset \widehat{G}$ and $r>0$, we define open sets $B_{A}(r) \subset G$ and $B_{A}^{*}(r) \subset L(G)$ by

$$
\begin{aligned}
& B_{A}(r)=\left\{g \in G \mid d_{A}(e, g)<r\right\}, \\
& B_{A}^{*}(r)=\left\{p \in L(G) \mid d_{A}^{*}(0, p)<r\right\} .
\end{aligned}
$$

Suppose $\rho$ is an endomorphism action of a semigroup $\Gamma$ on a solenoid $G$ and $A$ is a $\rho$-basis of $\widehat{G}$. Our next proposition shows that expansiveness of $\rho$ and existence of non-trivial bounded $\rho$-orbits in $L(G)$ can be characterized in terms of the metrics $d_{A}$ and $d_{A}^{*}$, respectively.

Proposition 4.3. Let $\Gamma$ be a discrete semigroup and $\rho$ be an endomorphism action of $\Gamma$ on a solenoid $G$. Let $A$ be a $\rho$-basis of $\widehat{G}$. Then we have the following:

1) $\rho$ is expansive if and only if $B_{A}(\epsilon)=\{e\}$ for some $\epsilon>0$.

2) $\rho_{e}$ has a non-trivial bounded orbit if and only if $B_{A}^{*}(C) \neq\{0\}$ for some $C>0$. 
Proof. 1) Suppose $B_{A}(\epsilon)=\{e\}$ for some $\epsilon>0$. We choose a finite set $F \subset A$ such that $A=\bigcup_{\gamma} \widehat{\rho}(\gamma)(F)$. Note that for any $g$ in $G$, the $\rho$-orbit of $g$ is contained in $B_{F}(\epsilon)$ only if $g \in B_{A}(\epsilon)$. Hence $B_{F}(\epsilon) \subset G$ is an expansive neighborhood of $e$ for the action $\rho$.

On the other hand suppose $U \subset G$ is an expansive neighborhood of $e$. We choose a finite set $F \subset A$ such that $A=\bigcup_{\gamma} \widehat{\rho}(\gamma)(F)$. Since $A$ generates $\widehat{G}$ as an abelian group, it follows that $B_{F}(\epsilon) \subset U$ for some $\epsilon>0$. Now it is easy to see that

$$
B_{A}(\epsilon)=\bigcap_{\gamma} \rho(\gamma)^{-1}\left(B_{F}(\epsilon)\right) \subset \bigcap_{\gamma} \rho(\gamma)^{-1}(U)=\{e\} .
$$

2) Suppose $p$ is a non-zero element of $L(G)$ such that the $\rho_{e}$-orbit of $p$ is bounded. We fix an element $\phi$ in $\widehat{G}$ and observe that

$$
\left\{q(\phi) \mid q \in \rho_{e}(\Gamma)(p)\right\}=\{p(\psi) \mid \psi \in \widehat{\rho}(\Gamma)(\phi)\} .
$$

Since $q \mapsto q(\phi)$ is a continuous map from $L(G)$ to $\mathbb{R}$ and the $\rho_{e}$-orbit of $p$ is bounded, the right-hand side is a bounded subset of $\mathbb{R}$. Hence $p$, restricted to the $\widehat{\rho}$-orbit of $\phi$, is bounded. Since $\phi$ is arbitrary and $A$ is a union of finitely many $\widehat{\rho}$-orbits, we conclude that $\left.p\right|_{A}$ is bounded i.e. $p \in B_{A}^{*}(C)$ for some $C>0$.

Conversely, suppose $p$ is an element of $B_{A}^{*}(C)$ for some $C>0$. We fix an element $\phi$ in $\widehat{G}$. Since $A$ generates $\widehat{G}$ as an abelian group, there exists a positive integer $l$ and $a_{1}, \ldots, a_{l}$ in $A$ such that $\phi=a_{1}+\cdots+a_{l}$. Since $A$ is invariant under $\widehat{\rho}$, every element in the $\widehat{\rho}$-orbit of $\phi$ can be written as a sum of $l$ elements of $A$. Hence $|p(\psi)|<l C$ for all $\psi$ in the $\widehat{\rho}$-orbit of $\phi$ i.e. $|q(\phi)|<l C$ for all $q$ in the $\rho_{e^{-o r b i t}}$ of $p$. Since $\phi$ is arbitrary, this implies that the $\rho_{e}$-orbit of $p$ is bounded.

4.3. Actions of finitely generated semigroups. As remarked earlier, for an arbitrary solenoid $G$, the map $E: L(G) \rightarrow G$ need not be surjective. However we will prove the following result which will be a crucial step in the proof of Theorem B.

Theorem 4.4. Let $\Gamma$ be a finitely generated discrete semigroup and let $\rho$ be an endomorphism action of $\Gamma$ on a solenoid $G$. Let $A$ be a $\rho$-basis of $\widehat{G}$. Then for any $C>0$ there exists an $\epsilon>0$ such that $B_{A}(\epsilon) \subset E\left(B_{A}^{*}(C)\right)$.

Note that this theorem, together with Propositions 4.1, 4.2 and 4.3, implies Theorem $\mathrm{B}$ when $\Gamma$ is finitely generated.

Remark. Earlier we have observed that $E$ is a continuous homomorphism and the kernel of $E$ is discrete. Hence the above theorem implies that the map $E$, restricted to $L(G)_{A}$, is a local homeomorphism if $L(G)_{A}$ and $G$ are equipped with the topologies induced by $d_{A}^{*}$ and $d_{A}$, respectively.

Before beginning the proof of Theorem 4.4 we introduce a few notations.

Let $H$ be a discrete abelian group and $A$ is a finite subset of $H$. Then by $\mathbf{Q}(A)$ we denote the set of all $h \in H$ which satisfies a linear equation of the form

$$
n_{0} h=\sum_{j=1}^{r} n_{j} a_{j},
$$

where $a_{1}, \ldots, a_{r}$ are elements of $A$ and $n_{0}, n_{1}, \ldots, n_{r}$ are integers with $n_{0} \neq 0$. Also for all $k>0$, by $\mathbf{Q}_{k}(A)$ we denote the set of all $h \in H$ which satisfies a linear equation as above with $\left|n_{0}\right|+\left|n_{1}\right|+\cdots+\left|n_{r}\right| \leq k$. A set $A \subset H$ is said to be 
$k$-regular if there exists an increasing sequence of finite sets $A_{1} \subset A_{2} \subset \cdots \subset A$ satisfying

$$
\bigcup_{i=1}^{\infty} A_{i}=A, \quad A_{n} \subset \mathbf{Q}_{k}\left(A_{n-1}\right) \quad \forall n \geq 2 .
$$

Now we show that if $\rho$ is an endomorphism action of a finitely generated semigroup on a solenoid $G$, then any $\rho$-basis is $k$-regular for some $k$.

Proposition 4.5. Let $\Gamma$ be a finitely generated semigroup and let $\rho$ be an endomorphism action of $\Gamma$ on a solenoid $G$. Then any $\rho$-basis of $\widehat{G}$ is $k$-regular for some $k>0$.

Proof. Let $A$ be a $\rho$-basis of $\widehat{G}$. Since $A$ generates $\widehat{G}$ as an abelian group and $\widehat{G}$ has finite rank, there exists a finite set $F_{1} \subset A$ such that $\mathbf{Q}\left(F_{1}\right)=\widehat{G}$. Also, $A=\bigcup_{\gamma} \widehat{\rho}(\gamma)\left(F_{2}\right)$ for some finite set $F_{2} \subset A$. We define $F=F_{1} \cup F_{2}$. Let $S \subset \Gamma$ be finite set which generates $\Gamma$ as a semigroup. Since $\mathbf{Q}(F)=\widehat{G}$, for any $\gamma$ in $S$ and $a_{0}$ in $F, \widehat{\rho}(\gamma)\left(a_{0}\right)$ satisfies a linear equation of the form

$$
n_{0} \cdot \widehat{\rho}(\gamma)\left(a_{0}\right)=\sum_{j=1}^{r} n_{j} a_{j}
$$

where $a_{1}, \ldots, a_{r}$ are elements of $F$ and $n_{0}, n_{1}, \ldots, n_{r}$ are integers with $n_{0} \neq 0$. Since $S$ is finite, it follows that there exists a positive integer $k$ such that $\widehat{\rho}(\gamma)(F) \subset$ $\mathrm{Q}_{k}(F)$ for all $\gamma$ in $S$. For $n \geq 1$ we define $S_{n} \subset \Gamma$ and $A_{n} \subset A$ by

$$
S_{n}=\left\{\gamma_{1} \cdots \gamma_{n} \mid \gamma_{1}, \ldots, \gamma_{n} \in S\right\}, \quad A_{n}=\bigcup_{\gamma \in S_{n}} \widehat{\rho}(\gamma)(F)
$$

Let $\gamma_{n}$ be any element of $S_{n}$. We choose $\gamma_{n-1} \in S_{n-1}$ and $\gamma_{0} \in S$ such that $\gamma_{n}=\gamma_{n-1} \cdot \gamma_{0}$. Since $\widehat{\rho}\left(\gamma_{0}\right)(F) \subset \mathbf{Q}_{k}(F)$, we see that

$$
\widehat{\rho}\left(\gamma_{n}\right)(F) \subset \widehat{\rho}\left(\gamma_{n-1}\right)\left(\mathbf{Q}_{k}(F)\right) \subset \mathbf{Q}_{k}\left(\widehat{\rho}\left(\gamma_{n-1}\right)(F)\right) .
$$

This shows that $A_{n} \subset \mathbf{Q}_{k}\left(A_{n-1}\right)$ for all $n \geq 2$. Since $S$ generates $\Gamma$, it is easy to see that $\bigcup A_{i}=\widehat{\rho}(\Gamma)(F)=A$.

Now we turn to the proof of Theorem 4.4 We will use the following lemma.

Lemma 4.6. Let $G$ be a solenoid and let $F$ be a finite subset of $\widehat{G}$. Then for any $C>0$ there exists an $\epsilon>0$ such that for any $g \in B_{F}(\epsilon)$ there exists $p$ in $B_{F}^{*}(C)$ satisfying $\phi(g)=\phi \circ E(p)$ for all $\phi$ in $F$.

Proof. Let $\widehat{H} \subset \widehat{G}$ be the subgroup generated by $F$ and let $H$ be the dual of $\widehat{H}$. Since $\mathbb{R}$ is divisible, any homomorphism from $\widehat{H}$ to $\mathbb{R}$ can be extended to a homomorphism from $\widehat{G}$ to $\mathbb{R}$. Therefore it is enough to consider the case when $G=H$. In that case there exists $n \geq 1$ and an isomorphism $\theta: G \rightarrow \mathbb{T}^{n}$ such that $\exp \circ \mathrm{d} \theta=\theta \circ E$. Since $B_{F}^{*}(C)$ is an open subset of $L(G)$ and exp $: L\left(\mathbb{T}^{n}\right) \rightarrow$ $\mathbb{T}^{n}$ is a local homeomorphism, it is easy to see that $E\left(B_{F}^{*}(C)\right)$ contains an open neighborhood of $e$ in $G$. Since $F$ generates $\widehat{G}$, there exists an $\epsilon>0$ such that $B_{F}(\epsilon) \subset E\left(B_{F}^{*}(C)\right)$. This proves the lemma. 
Proof of Theorem 4.4. Suppose $A$ is a $\rho$-basis of $\widehat{G}$. By Proposition $4.5 A$ is $k$ regular for some $k>0$. Clearly without loss of generality we may assume that $C<1 / k$. Let $A_{1} \subset A_{2} \subset \cdots \subset A$ be an increasing sequence of finite sets such that

$$
\bigcup_{i} A_{i}=A, \quad A_{n} \subset \mathbf{Q}_{k}\left(A_{n-1}\right) \quad \forall n \geq 2 .
$$

Applying Lemma 4.6 we choose a positive $\epsilon<C$ such that for all $h \in B_{A_{1}}(\epsilon)$ there exists a $q \in B_{A_{1}}^{*}(C)$ satisfying $\chi(E(q))=\chi(h)$ for all $\chi$ in $A_{1}$. Let $g$ be an element of $B_{A}(\epsilon)$. We choose a $p$ in $B_{A_{1}}^{*}(C)$ such that $\chi(E(p))=\chi(g)$ for all $\chi$ in $A_{1}$. We claim that

$$
|p(\chi)|<C, \chi(E(p))=\chi(g) \quad \forall \chi \in A .
$$

By our choice of $p$ this is true for all $\chi$ in $A_{1}$. Suppose this holds for all $\chi$ in $A_{n}$. Let $a_{0}$ be an element of $A_{n+1}$. We choose integers $n_{0}, n_{1}, \ldots, n_{r}$ and $a_{1}, \ldots, a_{r} \in A_{n}$ such that $n_{0} \neq 0,\left|n_{0}\right|+\left|n_{1}\right|+\cdots+\left|n_{r}\right| \leq k$ and

$$
n_{0} a_{0}=\sum_{j=1}^{r} n_{j} a_{j}
$$

Since $g \in B_{A}(\epsilon)$, there exists $\alpha \in \mathbb{R}$ such that $|\alpha|<\epsilon<C$ and $e^{2 \pi i \alpha}=a_{0}(g)$. Now from the above equation it is easy to see that

$$
e^{2 \pi i n_{0} \alpha}=a_{0}(g)^{n_{0}}=\prod_{1}^{r} a_{i}(g)^{n_{i}} .
$$

Since $a_{1}, \ldots, a_{r} \in A_{n}$, it follows that

$$
e^{2 \pi i n_{0} p\left(a_{0}\right)}=\prod_{1}^{r} e^{2 \pi i n_{j} p\left(a_{j}\right)}=\prod_{1}^{r} a_{i} \circ E(p)^{n_{i}}=\prod_{1}^{r} a_{i}(g)^{n_{i}} .
$$

Therefore $e^{2 \pi i n_{0}\left(\alpha-p\left(a_{0}\right)\right)}=0$ i.e. $n_{0}\left(\alpha-p\left(a_{0}\right)\right) \in \mathbb{Z}$. On the other hand

$$
\left|n_{0}\left(\alpha-p\left(a_{0}\right)\right)\right| \leq n_{0}|\alpha|+\left|p\left(n_{0} a_{0}\right)\right| \leq n_{0} C+\sum_{1}^{r} n_{i}\left|p\left(a_{i}\right)\right| .
$$

Now applying our induction hypothesis we see that

$$
\left|n_{0}\left(\alpha-p\left(a_{0}\right)\right)\right| \leq C \sum_{j=1}^{r}\left|n_{j}\right|<C k<1 .
$$

Hence $\alpha-p\left(a_{0}\right)=0$, which implies that $\left|p\left(a_{0}\right)\right|=|\alpha|<C$ and $a_{0}(E(p))=$ $e^{2 \pi i \alpha}=a_{0}(g)$. This proves the claim. Since $A$ generates $\widehat{G}$ as an abelian group, from the above claim we deduce that for any $g \in B_{A}(\epsilon)$ there exists a $p \in B_{A}^{*}(C)$ such that $E(p)=g$. This completes the proof.

4.4. Proof of Theorem B. Now we prove Theorem B for an endomorphism action $\rho$ of an arbitrary semigroup $\Gamma$ on a solenoid $G$. If $(G, \rho)$ is expansive, then from Proposition 4.1 and Proposition 4.2 it follows that a) and b) are satisfied. Conversely, suppose that conditions a) and b) are satisfied. By Lemma 3.1 there exists a finitely generated subsemigroup $\Gamma_{0}$ of $\Gamma$ such that for all $p$ in $L(G)$, the $\Gamma_{0}$-orbit of $p$ under the action $\rho_{e}$ is unbounded. We construct an endomorphism action $(H, \sigma)$ of $\Gamma_{0}$ as follows:

Since $\widehat{G}$ has finite rank, there exists a finite set $F_{1} \subset \widehat{G}$ such that $\mathbf{Q}\left(F_{1}\right)=\widehat{G}$. Also, from a) it follows that there exists a finite set $F_{2} \subset \widehat{G}$ such that the set 
$\bigcup_{\gamma} \widehat{\rho}(\gamma)\left(F_{2}\right)$ generates $\widehat{G}$ as an abelian group. We define $F=F_{1} \cup F_{2}$. Let $\widehat{H} \subset \widehat{G}$ be the subgroup generated by the set

$$
A_{0}=\bigcup_{\gamma \in \Gamma_{0}} \widehat{\rho}(\gamma)(F)
$$

and let $\widehat{\sigma}$ be the $\Gamma_{0}$-action on $\widehat{H}$ defined by $\widehat{\sigma}(\gamma)=\left.\widehat{\rho}(\gamma)\right|_{\widehat{H}}$. Let $H$ denote the dual of $\widehat{H}$ and let $\sigma$ denote the endomorphism action of $\Gamma_{0}$ on $H$ which is the dual of $\widehat{\sigma}$.

Claim. $(H, \sigma)$ is expansive.

Since $\widehat{G}=\mathbf{Q}(F) \subset \mathbf{Q}(\widehat{H})$, it follows that the restriction map $\left.p \mapsto p\right|_{\widehat{H}}$ is a $\Gamma_{0^{-}}$ equivariant linear isomorphism from $L(G)$ onto $L(H)$. This implies that for every non-zero $q \in L(H)$, the $\sigma_{e}$-orbit of $q$ is unbounded in $L(H)$. Also it is easy to see that $A_{0}$ is a $\sigma$-basis of $\widehat{H}$. Now applying Theorem 4.4 and Proposition 4.3 we see that $(H, \sigma)$ is expansive, which proves the claim.

Let $V$ be an expansive neighborhood of $e$ in $H$. Let $i: \widehat{H} \rightarrow \widehat{G}$ be the inclusion map and let $\pi: G \rightarrow H$ be the dual of $i$. We claim that $\pi^{-1}(V)$ is an expansive neighborhood for the action $\rho$. To see this we choose any $g$ in $G$ such that the $\rho$-orbit of $g$ is contained in $\pi^{-1}(V)$. It is easy to see that for any $\gamma \in \Gamma_{0}$,

$$
\sigma(\gamma) \circ \pi(g)=\pi \circ \rho(\gamma)(g) .
$$

Therefore the $\sigma$-orbit of $\pi(g)$ is contained in $V$. Since $V$ is an expansive neighborhood for $\sigma$, this implies that $\pi(g)=e$. In particular $\phi(g)=1$ for all $\phi$ in $F$. For any $\gamma$ in $\Gamma$, replacing $g$ by $\rho(\gamma)(g)$ and applying the same argument we see that

$$
\widehat{\rho}(\gamma)(\phi)(g)=\phi \circ \rho(\gamma)(g)=1 \quad \forall \gamma \in \Gamma, \phi \in F .
$$

Since $A=\bigcup_{\gamma \in \Gamma} \widehat{\rho}(\gamma)(F)$ generates $\widehat{G}$, it follows that $g=e$. This completes the proof.

Remark. Let $\Gamma$ be the group of positive rational numbers under multiplication, $\mathbb{Q}$ be the group of rational numbers under addition and $\widehat{\rho}$ be the action of $\Gamma$ on $\mathbb{Q}$ defined by $\widehat{\rho}(\gamma)(x)=\gamma \cdot x$. Let $\rho$ be the dual action of $\Gamma$ on $\widehat{\mathbb{Q}}$. Then from Theorem B it follows that $(\widehat{\mathbb{Q}}, \rho)$ is expansive. On the other hand it is easy to see that for any finitely generated subgroup $\Gamma_{0} \subset \Gamma, \mathbb{Q}$ is not finitely generated as a $\mathbb{Z}\left(\Gamma_{0}\right)$ module. Hence no finitely generated subgroup $\Gamma_{0} \subset \Gamma$ acts expansively on $\widehat{\mathbb{Q}}$ under the action $\rho$. Since $\Gamma$ is abelian, this shows that analogues of Corollary 3.2 and Corollary 3.4 do not hold when $G$ is a solenoid.

\section{ACKNOWLEDGEMENT}

I thank the referee for comments and suggestions.

\section{REFERENCES}

1. N. Aoki and M. Dateyama, The relationship between algebraic numbers and expansiveness of automorphisms on compact abelian groups, Fund. Math., no 117, 21-35, 1983. MR 85f:22008

2. S. Bhattacharya, Orbit equivalence and topological conjugacy of affine actions on compact abelian groups, Monatsh. Math., 129, no. 2, 89-96, 2000. MR 2000m:37017

3. M. Boyle and D. Lind, Expansive subdynamics, Trans. Amer. Math. Soc. 349: 55-102, 1997. MR 97d:58115

4. M. Eisenberg, Expansive automorphisms of finite-dimensional vector spaces, Fund. Math., no 59, 307-312, 1966. MR 34:3279

5. N. Jacobson, Lectures in abstract algebra, Vol 2, Van Nostrand, 1953. MR 14:837e 
6. A. Katok and K. Schmidt, The cohomology of expansive $\mathbb{Z}^{d}$-actions by automorphisms of compact abelian groups, Pacific J. Math, 170, no. 1, 105-142, 1995. MR 97b:22005

7. B. Kitchens and K. Schmidt, Automorphisms of compact groups, Ergodic Theory and Dynamical Systems 9: 691-735, 1989. MR 91g:22008

8. A. Knapp, Lie groups beyond an introduction, Progress in Mathematics, 140, Birkhäuser, 1996. MR 98b:22002

9. P. F. Lam, On expansive transformation groups, Trans. Amer. Math. Soc. 150: 131-138, 1970. MR 41:7661

10. W. M. Lawton, The structure of compact connected groups which admit an expansive automorphism, Recent advances in Topological Dynamics, Lecture Notes in Mathematics, Springer-Verlag, 1973. MR 52:11873

11. D. Lind and K. Schmidt, Homoclinic points of algebraic $\mathbb{Z}^{d}$-actions, J. Amer. Math. Soc. 12, no. 4, 953-980, 1999. MR 2000d:37002

12. L. S. Pontryagin, Topological groups, Translated from the second Russian edition by Arlen Brown Gordon and Breach Science Publishers, Inc., 1966. MR 34:1439

13. K. Schmidt, Automorphisms of compact abelian groups and affine varieties, Proc. London Math. Soc. 61: 480-496, 1990. MR 91j:28015

14. _ Dynamical systems of algebraic origin, Progress in Mathematics, 128, Birkhäuser Verlag, Basel, 1995. MR 97c:28041

School of Mathematics, Tata Institute of Fundamental Research, Mumbai 400005, INDIA

E-mail address: siddhart@math.tifr.res.in 